\title{
Task-related deactivation and functional connectivity of the subgenual cingulate cortex in major depressive disorder
}

\author{
Christopher G. Davey ${ }^{1,2}$, Murat Yücel ${ }^{2}$, Nicholas B. Allen ${ }^{1,3}$ and Ben J. Harrison ${ }^{2}$ \\ ${ }^{1}$ Orygen Youth Health Research Centre, The University of Melbourne, Melbourne, VIC, Australia \\ ${ }^{2}$ Melbourne Neuropsychiatry Centre, Department of Psychiatry and Melbourne Health, The University of Melbourne, Melbourne, VIC, Australia \\ ${ }^{3}$ Department of Psychological Sciences, The University of Melbourne, Melbourne, VIC, Australia
}

\section{Edited by:}

Alex Fornito, The University of

Melbourne, Australia

Reviewed by:

Amit Anand, Indiana University, USA

Jorge Almeida, University of

Pittsburgh, USA

\section{*Correspondence:}

Christopher G. Davey, Orygen Youth Health Research Centre, The University of Melbourne, Parkville, VIC 3052, Australia.

e-mail: c.davey@unimelb.edu.au
Background: Major depressive disorder is associated with functional alterations in activity and resting-state connectivity of the extended medial frontal network. In this study we aimed to examine how task-related medial network activity and connectivity were affected in depression. Methods: 18 patients with major depressive disorder, aged 15- to 24-years-old, were matched with 19 healthy control participants. We characterized taskrelated activations and deactivations while participants engaged with an executive-control task (the multi-source interference task, MSIT). We used a psycho-physiological interactions approach to examine functional connectivity changes with subgenual anterior cingulate cortex. Voxel-wise statistical maps for each analysis were compared between the patient and control groups. Results: There were no differences between groups in their behavioral performances on the MSIT task, and nor in patterns of activation and deactivation. Assessment of functional connectivity with the subgenual cingulate showed that depressed patients did not demonstrate the same reduction in functional connectivity with the ventral striatum during task performance, but that they showed greater reduction in functional connectivity with adjacent ventromedial frontal cortex. The magnitude of this latter connectivity change predicted the relative activation of task-relevant executive-control regions in depressed patients. Conclusion: The study reinforces the importance of the subgenual cingulate cortex for depression, and demonstrates how dysfunctional connectivity with ventral brain regions might influence executive-attentional processes.

Keywords: major depressive disorder, cognition, anterior cingulate cortex, striatum, default mode network, connectivity, fMRI, adolescence

\section{INTRODUCTION}

Major depressive disorder is characterized by symptoms in affective, somatic, and cognitive domains. The diversity of symptoms provides an indication that the illness arises from systemic alterations in brain function, and not from specific regional dysfunction. The brain system that has been most consistently implicated in the pathophysiology of depression is the extended medial prefrontal network (or "medial network") - a set of ventrally located brain regions that includes ventromedial frontal cortex, anterior and posterior cingulate cortex, striatum, amygdala, and thalamus (Price and Drevets, 2010). The abnormal function of these regions has been linked to key symptoms of depression such as low mood, anhedonia, and self-related disturbances (Keedwell et al., 2005; Grimm et al., 2009; Sheline et al., 2009). In addition to these disturbances, impairments of goal-directed cognitive processes are common in patients with depression, who frequently report problems with sustained attention and concentration (Gotlib and Joormann, 2010). By and large such processes are thought to reflect disturbances in the activity of dorsal frontoparietal brain regions, including the so-called "executive-control network" (Seeley et al., 2007). While both networks interact dynamically in the service of goal-directed behavior (Sridharan et al., 2008; Spreng et al., 2010), disturbances of the executive-control network are hypothesized to be secondary to medial network alterations in depressed patients (Price and Drevets, 2010). However, the putative mechanisms by which medial network activity may influence the engagement of executive-control processes in depression have not been well characterized.

The medial network, an essentially anatomical concept, shows considerable overlap with the hypothesized "default mode network," with both networks including as core components the ventromedial prefrontal cortex and ventral and posterior regions of the cingulate cortex. The default mode network was first described when it was observed that these regions, together with temporoparietal regions, showed greater metabolic activity when a person was at rest compared to when they were engaged in demanding cognitive tasks (Ghatan et al., 1995; Shulman et al., 1997; Raichle et al., 2001). This observed rest-task difference was termed "deactivation" because early imaging studies were primarily focused on "activation" to cognitive-attentional stimuli (Buckner et al., 2008), and we use the term in that sense here: to refer to brain activity that is reduced during task engagement compared to rest. It was subsequently noted that the default mode network showed functionally correlated activity during extended periods of continuous rest - while a person was engaged in "stimulus independent thought" (Greicius et al., 2003; Fox et al., 2005). More 
recently, examination of functional connectivity during these two types of rest - blocks of rest interleaved within cognitive tasks, and extended continuous rest - has shown that, while minor differences are evident, they are qualitatively and quantitatively very similar (Fair et al., 2007b).

The extent to which resting-state activity becomes less prominent, or "deactivates," during the performance of cognitive tasks has been related to their specific levels of demand (McKiernan et al., 2003; Mayer et al., 2010; Harrison et al., 2011), and has been shown to correlate with individual differences in task reaction times and accuracy (Harrison et al., 2007; Anticevic et al., 2010; Sala-Llonch et al., 2011). In healthy people cognitive tasks have been shown to affect default mode network connectivity in two ways: firstly, connectivity between different regions of the default mode network remains relatively consistent during task performance (Hampson et al., 2006; Fransson and Marrelec, 2008; Harrison et al., 2008; Bluhm et al., 2011); and secondly, there is reduced functional connectivity between default and non-default mode network regions (Bluhm et al., 2011).

The above observations may be relevant to depression, in which resting-state alterations in activity and connectivity of ventral regions of the anterior cingulate cortex (ACC) have been a frequent finding. The subgenual ACC, in particular, has been reported to show increased resting-state activity in nuclear imaging studies of depressed patients (Drevets, 1999; Dunn et al., 2002), and stronger functional connectivity between the subgenual ACC and other regions of the medial network have been shown using functional magnetic resonance imaging (Greicius et al., 2007; Hamilton et al., 2011; Davey et al., 2012). A recent study reported increased functional connectivity of dorsomedial frontal cortex (in a region the authors labeled the "dorsal nexus") with subgenual ACC, dorsolateral frontal cortex, and precuneus (Sheline et al., 2010). Alterations in connectivity with the dorsal ACC - an important region for cognitive control processes - have also been reported, with demonstration of reduced connectivity with striatum, amygdala, and medial thalamus (Anand et al., 2005). During performance of cognitive tasks depressed patients have shown broadly reduced deactivation of the ventral ACC and extended medial frontal cortex (Harvey et al., 2005; Wagner et al., 2006). Connectivity changes in medial network regions during cognitive task performance, however, have not previously been reported for depressed patients, despite evidence that such changes may underlie task-related cognitive impairment in other psychiatric disorders (Whitfield-Gabrieli et al., 2009).

The goal of the current study was therefore to examine whether depressed patients showed differences in deactivation, and in functional connectivity of the subgenual ACC, during cognitive task performance. We selected the subgenual ACC as a region-ofinterest because of its frequent implication in depression (Hamani et al., 2011) - including being the site for the placement of electrical leads in deep-brain-stimulation (DBS) treatment of the illness (Lozano et al., 2008). The subgenual ACC has previously been shown to be more strongly connected with the default mode network in depressed compared to control participants (Greicius et al., 2007), suggesting the possibility of altered changes in connectivity during engagement with cognitive tasks that could be expected to deactivate the network. Our predictions were that depressed participants would show less deactivation of default mode regions during cognitive task performance, in keeping with other studies. Secondly, we expected that functional connectivity with the subgenual ACC would be distinctly modulated in depressed patients when comparing rest and task performance periods. To assess this, participants were examined with functional MRI while they performed the multi-source interference task (MSIT; Bush and Shin, 2006). The MSIT is a well-validated executive-control paradigm that evokes robust activation of dorsal frontoparietal regions, and corresponding deactivation of the default mode network (Bush and Shin, 2006; Harrison et al., 2007; Yucel et al., 2007). In order to reliably assess changes in deactivation and subgenual ACC functional connectivity from a resting baseline, we developed a modified version of the MSIT that interleaved specific rest-fixation periods between each task block. We employed an analysis based on psycho-physiological interactions (PPI; Friston et al., 1997) in order to identify changes in functional connectivity of the subgenual ACC between the resting and task performance states.

\section{MATERIALS AND METHODS PARTICIPANTS}

Depressed participants were recruited for the study from Orygen Youth Health, a public mental health service for young people in Melbourne, Australia. Patients were between 15 and 24 years of age, and had major depressive disorder determined by Structured Clinical Interview for DSM-IV (SCID; First et al., 1997). Patients were included from an age range that extended from the middle teenage years to early adulthood, which accords with a clinical focus on youth mental health (McGorry, 1998), and is consistent with our current understanding of the continuities in brain and social development through this period (Davey et al., 2008). Patients did not meet the criteria for psychotic disorder, substance dependence disorder, pervasive developmental disorder, or intellectual disability; and were not excluded if they were taking antidepressant medication. The depressed participants were successfully matched with a group of control participants on age, gender, and full-scale IQ (Table 1). The control participants were recruited via advertisement placed in a daily metropolitan newspaper, and had no history of mental illness, as determined by SCID. The participants (and their parents if they were younger than 18 years of age) provided their informed consent to participate in the study, which was approved by the local research and ethics committees. Imaging data from one control participant and one depressed participant were excluded due to excessive head movements during scanning (see further), resulting in a control group of 19 participants and a depressed group of 18 participants.

The illness characteristics of the depressed participants reflected their relatively young age, and their recruitment via a public mental health service, which treats young people with relatively severe illness. The mean Beck Depression Inventory (BDI-II) score for the depressed participants of 34.4 indicates that their illnesses were at the severe end of the illness spectrum (Beck et al., 1996). Nine of the depressed participants were being treated with antidepressant medication: fluoxetine $(n=4)$, citalopram $(n=2)$, and venlafaxine $(n=3)$. 
Table 1 | Characteristics of the control and depressed participants.

\begin{tabular}{lll}
\hline Participant variable & $\begin{array}{l}\text { Mean (SD), median } \\
\text { or \% (number) }\end{array}$ & $\begin{array}{l}\text { Mean (SD), median } \\
\text { or \% (number) }\end{array}$ \\
\hline Age & $19.9(2.7)$ & $18.9(2.2)$ \\
Female & $58 \%(11)$ & $61 \%(11)$ \\
Full-scale IQ & $109.8(11.0)$ & $107.9(9.3)$ \\
BDI score & $3.1(3.6)$ & $34.4(12.6)$ \\
First episode of depression & - & $50.0 \%(9)$ \\
Median length of episode & - & 10.5 months \\
Comorbid anxiety disorder & - & $33.3 \%(6)$ \\
Antidepressant medication & - & $50.0 \%(9)$ \\
Median length of treatment & - & 4 months \\
\hline
\end{tabular}

\section{TASK DESCRIPTION}

Each participant performed a modified block-design version of the MSIT, which involved responding to eight 30-s task blocks of congruent (i.e., lower cognitive demand) and incongruent (i.e., higher cognitive demand) stimulus trials. During both task conditions, participants were instructed to indicate the identity (not the position) of a visually presented target number in a three-digit sequence corresponding to learned button box associations using the index (1), middle (2), and ring (3) fingers. During congruent trials the target number always matched its appropriate button box position and was flanked by zeros (e.g., "0 20 "). During incongruent trials, the target never matched its appropriate position and was flanked by incongruent numbers (e.g., "3 11 1"). Each block consisted of 12 trials of either the congruent or incongruent stimulus conditions, with the stimulus presented for $2000 \mathrm{~ms}$ and an inter-stimulus interval of $500 \mathrm{~ms}$. As implemented by Harrison et al. (2011), each task block was interleaved with $15 \mathrm{~s}$ periods of rest-fixation. The main focus of our analysis was on comparison of the rest blocks with the cognitively demanding incongruent task blocks.

\section{IMAGE ACOUISITION AND PREPROCESSING}

A 3-T Siemens Magnetom Trio magnetic resonance scanner (Erlangen, Germany) was used to acquire whole-brain functional T2*-weighted echo-planar images (EPIs). Functional sequences consisted of gradient-recalled acquisition in the steady state [time of repetition (TR), $2400 \mathrm{~ms}$; time of echo (TE), $40 \mathrm{~ms}$; pulse angle, $90^{\circ}$ ) within a field of view of $210 \mathrm{~mm}$, with a $64 \times 64$-pixel matrix, and with a slice thickness of $3 \mathrm{~mm}$ (no inter-slice gap). Thirty-six interleaved slices were acquired parallel to the anterior-posterior commissure line, with particular attention paid to ensuring good coverage of ventral brain areas. Field maps were also acquired to correct for distortion caused by magnetic field inhomogeneities (TR, $400 \mathrm{~ms}$; short TE, $5.19 \mathrm{~ms}$; long TE, $7.65 \mathrm{~ms}$; pixel matrix, $64 \times 64$; slice thickness, $3 \mathrm{~mm}$ ). While completing the MSIT task 157 whole-brain EPIs were acquired for each participant. In addition, prior to performing the MSIT, each participant underwent a single 12.3-min resting-state sequence (they were instructed to relax, stay awake, and lie still with their eyes closed) with the acquisition of 307 EPIs. A high-resolution T1-weighted anatomical image was also acquired to aid co-registration of the functional sequences to standard neuroanatomical space. During scanning participants were provided with earphones to reduce scanner noise, and foam-rubber inserts were used to aid head stability.

Imaging data were transferred and processed on an Apple OSX platform running MATLAB version 7 (MathWorks, Natick, MA, USA), and using Statistical Parametric Mapping 8 (SPM8) ${ }^{1}$. Motion correction was performed by aligning each participant's time series to the first image using least-squares minimization and a six-parameter (rigid body) spatial transformation, and the field maps were used to unwarp the images. Participants' data were excluded from analysis if translation and rotation estimates were greater than $2 \mathrm{~mm}$ or $2^{\circ}$, respectively. Excessive movement resulted in the exclusion of data from one control participant and one depressed participant. The realigned and unwarped functional sequences were then coregistered to each participant's respective anatomical scan. Anatomical scans were segmented and spatially normalized to the International Consortium for Brain Mapping template using the unified segmentation approach, and the normalization parameters were applied to the coregistered functional images, which were resliced to $2 \mathrm{~mm}$ isotropic resolution. Functional images were smoothed with a 6-mm (full-width halfmaximum) Gaussian filter. All image sequences were routinely inspected for potential normalization artifacts.

\section{STATISTICAL ANALYSIS}

For each participant primary task regressors were created by specifying the onset and duration of each task block, followed by convolution with a canonical hemodynamic response function and its temporal derivative, and use of a high-pass filter set at $128 \mathrm{~s}$ to remove low-frequency drifts. Parameter estimates were calculated at each voxel using the general linear model and local autocorrelation correction. Second-level analysis identified voxels that showed greater activation in the incongruent compared to congruent task, and in the rest block compared to the incongruent task (i.e., that were deactivated during the task). To characterize any between-group differences we thresholded the statistical maps at $P<0.005$, and determined whether any clusters survived wholebrain cluster-wise correction $\left(P_{\mathrm{FDR}}<0.05\right)$ within broad global masks of relevant activated or deactivated brain regions (formed by global conjunction of the control and depressed participants, thresholded at $P<0.05$, uncorrected).

Psycho-physiological interactions were examined using seed regions of interest in the subgenual ACC. PPI analysis allows an examination of whether connectivity between brain regions differs in different psychological context. It assesses whether there is an interaction between correlated brain activations and the psychological task; in other words whether there is an influence of one brain region on another that is over-and-above correlated activations and deactivations produced by the task (Friston et al., 1997). In this study our focus was on how connectivity with the subgenual ACC changed between resting-state periods and performance of the incongruent MSIT condition. The location of the seeds was taken from Margulies et al.'s (2007) study of cingulate connectivity: spheres of $3.5 \mathrm{~mm}$ radius were created in left and right subgenual ACC $(x= \pm 5, y=25, z=-10)$. Time series

${ }^{1}$ http://www.fil.ion.ucl.ac.uk/spm/ 
were extracted for each seed; and the deconvolved time course, along with the task model (rest $>$ incongruent task), and their interaction, were entered into a first-level GLM analysis for each participant. The voxel-wise statistical maps calculated for each PPI were then included in group (second-level) random-effects analyses, adopting a $2 \times 2$ factorial model [group (control, patient) by hemisphere (right seed, left seed)]. Age and medication status were included as covariates.

Our primary interest was in characterizing how resting functional connectivity of the subgenual ACC changed in response to task performance in the two groups. We therefore limited our analysis to only those regions that showed significant functional connectivity with the subgenual ACC during resting-state conditions by masking our second-level PPI analyses with an independently derived resting-state functional connectivity map (i.e., from the 12-min resting-state scans from the same participants; reported in Davey et al., 2012). The map was created by including the resting-state time-courses for the subgenual ACC seeds and nuisance signals (white matter, cerebrospinal fluid, and global brain signal fluctuations) as predictors of interest and no interest, respectively, in whole-brain, linear regression analyses. Contrast images were generated for each participant by estimating the regression coefficient between all brain voxels and each seed's time series, separately for each hemisphere. These images were then included in group random-effects analyses, using a similar $2 \times 2$ factorial as above (detailed results of the analyses are to be presented elsewhere). The restingstate mask was created for the subgenual ACC by thresholding a conjunction map for control and depressed participants at voxel-wise $P_{\mathrm{FDR}}<0.05$, ensuring that the second-level PPI analyses were restricted to only those brain regions that showed significant resting-state correlations with the subgenual ACC in both the depressed and control groups. The mask consisted of the core components of the medial network, including ventral cingulate cortex, ventromedial frontal cortex, posterior cingulate cortex, striatum, and anterior thalamus. Analyses of within- and between-group PPI effects were thresholded at a voxel-wise $P<0.005$, and cluster-level probabilities were calculated using Monte Carlo simulation, as implemented by AlphaSim ${ }^{2}$

To investigate potential associations between subgenual ACC connectivity and other task-related brain and behavioral indices, we performed second-level GLM covariance analyses in each group that examined whether changes in connectivity predicted: (i) the relative magnitude of task-related activation of executivecontrol regions during the MSIT; and (ii) individual differences in task performance with regards to participants' reaction-time and accuracy scores. In the first analysis, the strengths of connectivity between the subgenual ACC and implicated regions of interest (see Results) were estimated by extracting the first eigenvariate of the voxels within each region-of-interest using SPM8. These values were then entered into the second-level model of task activation effects (incongruent task $>$ rest). In the next analysis, participants' performance scores were entered as covariates of interest into the

${ }^{2}$ http://afni.nimh.nih.gov/afni/doc/manual/AlphaSim. second-level model of task-related subgenual ACC connectivity changes (rest $>$ incongruent task). For the patient group only, we additionally examined direct associations between connectivity changes and clinical severity, as measured with the BDI. All analysis were thresholded at $P<0.005$ with whole-brain cluster-wise correction $(P<0.05)$.

\section{RESULTS}

The control and depressed groups performed the MSIT task similarly. They showed similar high rates of accuracy (controls: congruent $99.8 \%$, incongruent $97.5 \%$; depressed: congruent 99.6\%, incongruent 96.1\%; main effect of task: $F_{1,35}=22.6$, $P=<0.001$; main effect of group: $F_{1,35}=0.86, P=0.36$; interaction: $F_{1,35}=0.38, P=0.54$ ); and similar reaction times (controls: congruent $657 \mathrm{~ms}$, incongruent $1032 \mathrm{~ms}$; depressed: congruent $628 \mathrm{~ms}$, incongruent $1035 \mathrm{~ms}$; main effect of task: $F_{1,35}=372.8$, $P=<0.001$; main effect of group: $F_{1,35}=0.22, P=0.65$; interaction: $\left.F_{1,35}=0.64, P=0.43\right)$.

The MSIT paradigm led to robust activation (incongruent task > congruent task) of the bilateral dorsal frontoparietal cortex, dorsal anterior ACC, supplementary motor cortex, and other executive-control areas. Prominent task-related deactivations (rest $>$ incongruent task) were observed in the ventral and dorsal posterior cingulate cortex, precuneus and the ventromedial frontal cortex (Figure 1). When comparing the two groups directly, no significant differences in task-related activation or deactivation were observed, even at a lenient uncorrected minimum cluster size of 20 voxels. Further region-of-interest analysis of deactivations within the subgenual ACC seeds confirmed no differences between groups (main effect of group: $F_{1,35}=1.53, P=0.23$ ).

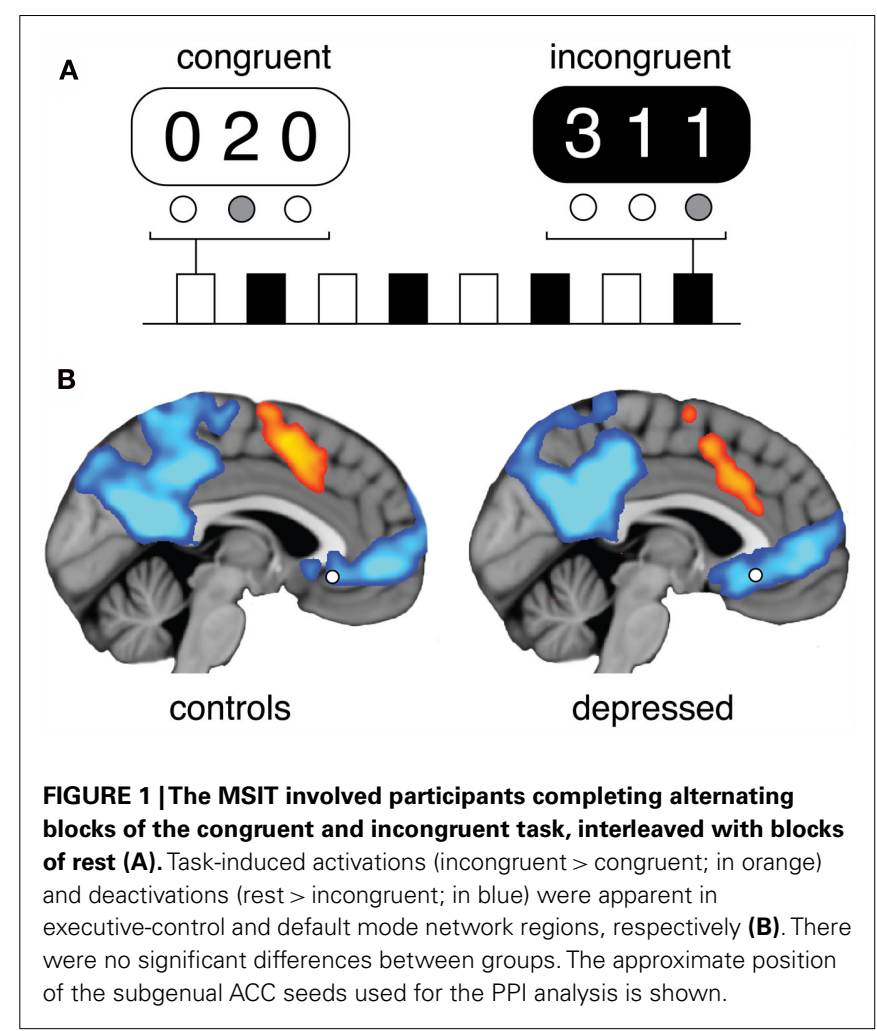


In our assessment of task-related functional connectivity, control participants demonstrated greater connectivity of the subgenual ACC with the right ventral caudate/nucleus accumbens during rest when compared to task performance; that is, task engagement was associated with a decreased in connectivity between the regions. Depressed participants, on the other hand, showed greater connectivity between the subgenual ACC and adjacent ventromedial frontal cortex regions at rest compared to task performance: task engagement was associated with a decrease in connectivity. Between-group comparisons confirmed that these taskrelated connectivity changes were significantly different between the healthy and depressed participants (Table 2; Figure 2).

No significant linear associations were observed between the aforementioned connectivity changes and participants' behavioral task performance (i.e., reaction-time and accuracy), nor with depression severity in the depressed participants. However, correlations were observed between the magnitude of task-induced reduction in connectivity with the ventromedial frontal cortex in MDD patients and task-related activation of the left superior parietal cortex [cluster size, 694 voxels; peak coordinate $\left(\begin{array}{lll}x & y & z\end{array}\right),-28$ -32 40; peak $Z$-score, 5.3; cluster-level $P_{F D R}=0.002$ ] and left dorsolateral frontal cortex [cluster size, 287 voxels; peak coordinate ( $x y z$ ), -48 26 26; peak $Z$-score, 4.4; cluster-level $P_{F D R}=0.11$; Figure 2]. That is, patients who demonstrated a more prominent decrease in connectivity between the subgenual ACC and ventromedial frontal cortex in the rest compared to task conditions also demonstrated more prominent task-related activations of left frontoparietal regions. No such significant associations were observed in control participants.

\section{DISCUSSION}

The aim of the study was to examine how medial network regions were affected by cognitive task performance in depression, both in terms of task-induced deactivations and functional connectivity changes. The results demonstrate that despite being similarly deactivated during the performance of the MSIT task, the subgenual ACC was distinctly modulated in its functional connectivity with other brain regions in depressed participants. Whereas a reduction in connectivity between this region and the ventral striatum was characteristic of the transition between rest and task performance in the control group, a reduction in connectivity with the ventromedial frontal cortex was characteristic of depression. Moreover, the magnitude of this latter connectivity change was found to predict the corresponding activation of task-related frontoparietal regions, suggesting a direct link between putative medial network alterations and executive-control processes in depression.

Medial network areas project prominently to ventral striatum (which is particularly a target of subgenual ACC) and to the medial edge of the caudate nucleus (Ferry et al., 2000; Beckmann et al., 2009). In healthy participants, cognitive task engagement was found to significantly decrease functional connectivity between the subgenual ACC and ventral striatum from its resting-baseline level, which is an observation that has not previously been reported (we are, in fact, not aware of any studies that have specifically investigated the effects of cognitive engagement on the functional connectivity of the subgenual ACC). Given that both regions are major components of the medial frontal network, which underlies mood and emotional states, it is possible that a decrease in their functional connectivity during task performance corresponds with reduced subjective awareness in these domains. This idea is consistent with a growing body of evidence that has associated the deactivation of default mode network regions during external task performance with reduced self-focused spontaneous cognition that is characteristic of wakeful resting conditions (Mason et al., 2007; Christoff et al., 2009; Andrews-Hanna et al., 2010; see also Harrison et al., 2011). Whether task-induced changes in subgenual ACC connectivity represent a reduction in awareness or experience of mood or emotional state requires further investigation.

Unlike healthy participants, depressed participants did not show any modulation of connectivity between the subgenual ACC and ventral striatum, suggesting that the functional coupling of these regions persisted between the resting and task conditions. The subgenual ACC and ventral striatum showed significant resting-state connectivity in both groups (as incorporated in our resting-state mask), and have not previously been reported to show resting-state connectivity alterations in depression. The finding is therefore most likely explained by connectivity differences during task performance. The striatum has previously been reported to show decreased activation to rewarding stimuli in both depressed patients (Pizzagalli et al., 2009) and those at risk of depression

Table 2 | Functional connectivity changes with subgenual ACC from rest to cognitive control task, thresholded at $P<0.005$.

\begin{tabular}{|c|c|c|c|c|}
\hline Brain region & Number of voxels in cluster & Peak Z-score & Peak voxel coordinates $(x y z)$ & Cluster-level probability* \\
\hline \multicolumn{5}{|l|}{ CONTROLS } \\
\hline R. Ventral striatum & 155 & 5.1 & $1616-6$ & $<0.01$ \\
\hline \multicolumn{5}{|l|}{ DEPRESSED } \\
\hline L. Medial frontal gyrus (BA 10) & 57 & 3.6 & $-1234-10$ & 0.01 \\
\hline \multicolumn{5}{|l|}{ CONTROLS > DEPRESSED } \\
\hline R. Ventral striatum & 52 & 3.4 & $1416-6$ & 0.01 \\
\hline \multicolumn{5}{|l|}{ DEPRESSED > CONTROL } \\
\hline L. Medial frontal gyrus (BA 10) & 61 & 4.4 & $-1634-8$ & $<0.01$ \\
\hline R. Medial frontal gyrus (BA 10) & 19 & 3.5 & $1238-10$ & $(0.06)$ \\
\hline
\end{tabular}

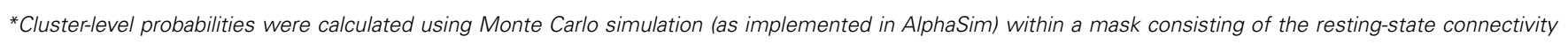
map for the subgenual ACC. 
A

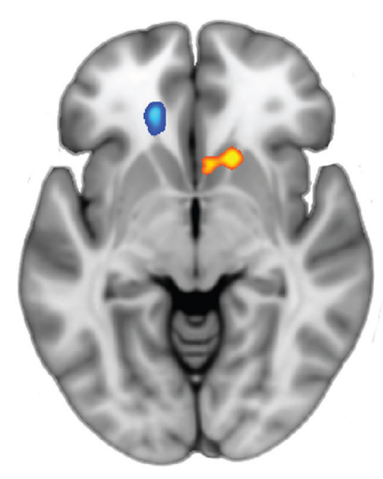

C

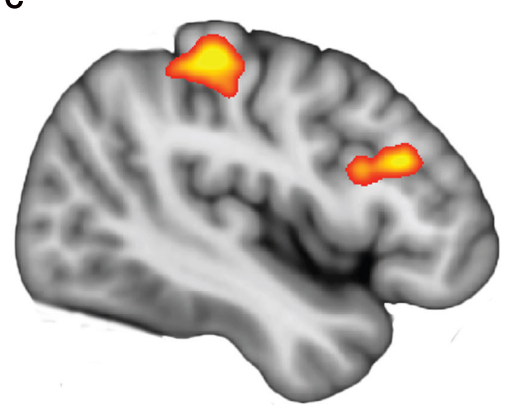

B

D

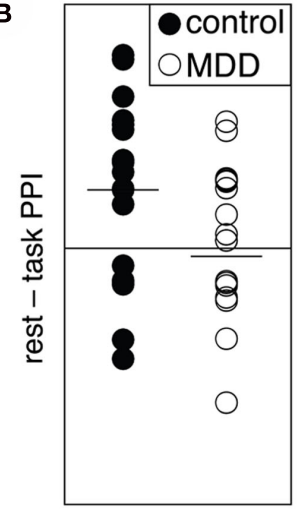

striatum

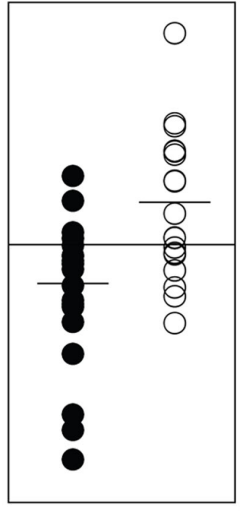

vmPFC

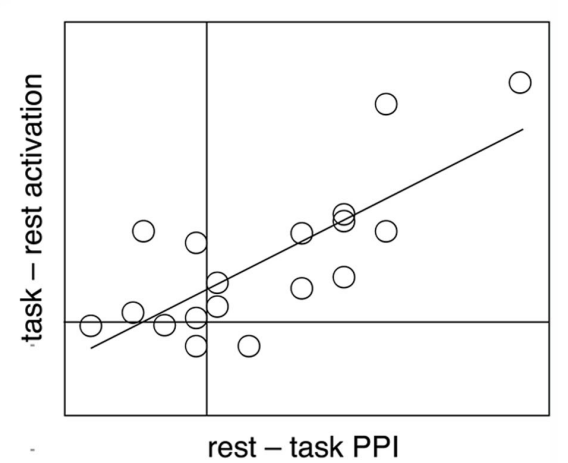

value indicates increased connectivity at rest compared to task (or reduced connectivity during task performance). For the depressed participants, the extent of the reduction in connectivity between the subgenual ACC and ventromedial frontal cortex predicted task-induced activations in left superior parietal cortex and left dorsolateral frontal cortex (C). The adjacent chart (D) illustrates the correlation, with the parameter values extracted from $3.5 \mathrm{~mm}$ spheres around the peak voxels from the ventromedial frontal cortex and superior parietal cortex.
(Gotlib et al., 2010). The results of this study suggest that this might in part be explained by depression-related impairment of task-induced connectivity changes with the subgenual ACC, whereby the altered connectivity influences the responsiveness of the ventral striatum to certain types of external stimulation. An examination of such interactions may usefully extend previous studies on reward processing impairments in depression.

While depressed participants did not show connectivity changes between subgenual ACC and striatum, they did show connectivity changes with adjacent ventromedial frontal cortex. Default mode network functional connectivity has been demonstrated to be robust and relatively consistent across task conditions in healthy participants (Hampson et al., 2006; Fransson and Marrelec, 2008; Harrison et al., 2008; Bluhm et al., 2011), and no differences in connectivity between the subgenual ACC and default mode regions were observed in control participants in this study. Not only did depressed participants show reduced connectivity between the subgenual ACC and ventromedial frontal cortex during task performance compared to rest, but the extent of the reduction showed correlation with greater activation of task-relevant executive-control regions. The fact that there were no differences between the groups in either behavioral performance or task-induced activations suggests that the connectivity changes may be functionally adaptive: that is, the connectivity changes allowed the depressed participants to perform the task in a manner similar to the control participants.

The lack of task-induced deactivation changes is in contrast to previous studies that have reported reduced deactivations in depressed participants performing cognitive tasks (Harvey et al., 2005; Wagner et al., 2005) - who, similarly to the findings of our study, showed no differences in behavioral performances. It is not clear why our study did not show the same deactivation differences, though one explanation is that our study included younger patients in early phases of illness, when cognitive impairment is not such a significant component (Grant et al., 2001; Kyte et al., 2005). Despite not showing differences in task-induced deactivations, the depressed participants did show different patterns of connectivity changes, which may presage changes in medial network deactivation and cognitive task performances later in the course of their illnesses. 
The study has its limitations. Our study sample included young people ranging in age from late adolescence to early adulthood; and while the age range is relatively narrow, it does encompass a developmental period over which significant brain maturational processes occur, including changes in brain connectivity These changes from childhood to adulthood include decreases in shortrange connections and increases in longer-range connections (Fair et al., 2007a; Supekar et al., 2009; Dosenbach et al., 2010) - including with cingulate regions (Kelly et al., 2009) - with increasing coherence in connectivity of the default mode network (Fair et al., 2008). Age was included as a covariate in our analyses, however, and is therefore unlikely to have had significant influence on the results. We included participants who were taking medication, reflective of the nature of the clinical group recruited for the study. Again, this was added as a covariate to the analyses to mitigate its influence on the current results. A further limitation of the study is inherent in the PPI methodology used. While PPI provides a relatively simple and robust way of investigating functional connectivity changes during task performance, it cannot provide information on the causal influence that one brain region exerts on another (or "effective connectivity"). The results of study could be usefully validated and extended with the use of a technique such as dynamic causal modeling (DCM;

\section{REFERENCES}

Anand, A., Li, Y., Wang, Y., Wu, J., Gao, S., Bukhari, L., Mathews, V. P., Kalnin, A., and Lowe, M. J. (2005). Activity and connectivity of brain mood regulating circuit in depression: a functional magnetic resonance study. Biol. Psychiatry 57, 1079-1088.

Andrews-Hanna, J. R., Reidler, J. S., Huang, C., and Buckner, R. L. (2010). Evidence for the default network's role in spontaneous cognition. J. Neurophysiol. 104, 322-335.

Anticevic, A., Repovs, G., Shulman, G. L., and Barch, D. M. (2010). When less is more: TPJ and default network deactivation during encoding predicts working memory performance. Neuroimage 49, 2638-2648.

Beck, A. T., Steer, R. A., and Brown, G. K. (1996). Manual for the Beck Depression Inventory-II. San Antonio: Psychological Corporation.

Beckmann, M., Johansen-Berg, H., and Rushworth, M. F. (2009). Connectivity-based parcellation of human cingulate cortex and its relation to functional specialization. $J$. Neurosci. 29, 1175-1190.

Bluhm, R. L., Clark, C. R., McFarlane, A. C., Moores, K. A., Shaw, M. E., and Lanius, R. A. (2011). Default network connectivity during a working memory task. Hum. Brain Mapp. 32, 1029-1035.

Buckner, R. L., Andrews-Hanna, J. R., and Schacter, D. L. (2008). The brain's default network: anatomy, function, and relevance to disease. Ann. N. Y. Acad. Sci. 1124, 1-38.

Bush, G., and Shin, L. M. (2006). The multi-source interference task: an fMRI task that reliably activates the cingulo-frontal-parietal cognitive/attention network. Nat. Protoc. 1, 308-313.

Christoff, K., Gordon, A. M., Smallwood, J., Smith, R., and Schooler, J. W. (2009). Experience sampling during fMRI reveals default network and executive system contributions to mind wandering. Proc. Natl. Acad. Sci. U.S.A. 106, 8719-8724.

Davey, C. G., Harrison, B. J., Yücel, M., and Allen, N. B. (2012). Regionally specific alterations in functional connectivity of the anterior cingulate cortex in major depressive disorder. Psychol. Med.

Davey, C. G., Yucel, M., and Allen, N. B. (2008). The emergence of depression in adolescence: development of the prefrontal cortex and the representation of reward. Neurosci. Biobehav. Rev. 32, 1-19.

Dosenbach, N. U., Nardos, B., Cohen, A. L., Fair, D. A., Power, J. D., Church, J. A., Nelson, S. M., Wig, G. S., Vogel, A. C., Lessov-Schlaggar, C. N., Barnes, K. A., Dubis, J. W., Feczko, E., Coalson, R. S., Pruett, J. R. J., Barch, D. M., Petersen, S. E., and Schlaggar, B. L. (2010). Prediction of individual brain maturity using fMRI. Science 329, 1358-1361.

Drevets, W. C. (1999). Prefrontal cortical-amygdalar metabolism in

Friston et al., 2003), which would be able to provide more information on what was driving the connectivity changes we have shown.

The study reinforces the importance of the extended medial prefrontal network for depression. It demonstrates how alterations in connectivity in the network, which mainly supports visceromotor function, might also influence cognitive-attentional processes. The reported dysfunction in cognitive and attentional processes that are often reported in depressed participants - and in the underlying frontoparietal brain regions that support them - might in fact be a secondary consequence of medial network dysfunction. The subgenual cingulate cortex is an important region for depression, and the results of the study provide further evidence for how dysfunction of subgenual ACC contributes to the illness.

\section{ACKNOWLEDGMENTS}

Dr. Davey is supported by a National Health and Medical Research Council (NHMRC) Early Career Fellowship (ID No. 628922), Dr. Harrison (ID No. 628509) is supported by an NHMRC Clinical Career Development Award, Dr. Yücel (ID No. 509345) is supported by an NHMRC Senior Research Fellowship (ID No. 1021973), and Dr. Allen is partially supported by a grant from the Colonial Foundation.

major depression. Ann. N. Y. Acad. Sci. 877, 614-637.

Dunn, R. T., Kimbrell, T. A., Ketter, T. A., Frye, M. A., Willis, M. W., Luckenbaugh, D. A., and Post, R. M. (2002). Principal components of the Beck Depression Inventory and regional cerebral metabolism in unipolar and bipolar depression. Biol. Psychiatry 51, 387-399.

Fair, D. A., Cohen, A. L., Dosenbach, N. U., Church, J. A., Miezin, F. M., Barch, D. M., Raichle, M. E., Petersen, S. E., and Schlaggar, B. L. (2008). The maturing architecture of the brain's default network. Proc. Natl. Acad. Sci. U.S.A. 105, 4028-4032.

Fair, D. A., Dosenbach, N. U., Church, J. A., Cohen, A. L., Brahmbhatt, S., Miezin, F. M., Barch, D. M., Raichle, M. E., Petersen, S. E., and Schlaggar, B. L. (2007a). Development of distinct control networks through segregation and integration. Proc. Natl. Acad. Sci. U.S.A. 104, 13507-13512.

Fair, D. A., Schlaggar, B. L., Cohen, A. L., Miezin, F. M., Dosenbach, N. U., Wenger, K. K., Fox, M. D., Snyder, A. Z., Raichle, M. E., and Petersen, S. E. (2007b). A method for using blocked and event-related fMRI data to study "resting state" functional connectivity. Neuroimage 35, 396-405.

Ferry, A. T., Ongur, D., An, X., and Price, J. L. (2000). Prefrontal cortical projections to the striatum in macaque monkeys: evidence for an organization related to prefrontal networks. J. Comp. Neurol. 425, 447-470.

First, M. B., Spitzer, R. L., Gibbon, M., and Williams, J. B. W. (1997). Structured Clinical Interview for DSM-IV Axis I Disorders (SCID-I). Washington: American Psychiatric Publishing.

Fox, M. D., Snyder, A. Z., Vincent, J. L., Corbetta, M., Van Essen, D. C., and Raichle, M. E. (2005). The human brain is intrinsically organized into dynamic, anticorrelated functional networks. Proc. Natl. Acad. Sci. U.S.A. 102, 9673-9678.

Fransson, P., and Marrelec, G. (2008). The precuneus/posterior cingulate cortex plays a pivotal role in the default mode network: evidence from a partial correlation network analysis. Neuroimage 42, 1178-1184.

Friston, K. J., Buechel, C., Fink, G. R., Morris, J., Rolls, E., and Dolan, R. J. (1997). Psychophysiological and modulatory interactions in neuroimaging. Neuroimage 6, 218-229.

Friston, K. J., Harrison, L., and Penny, W. (2003). Dynamic causal modelling. Neuroimage 19, 1273-1302.

Ghatan, P. H., Hsieh, J. C., WirsenMeurling, A., Wredling, R., Eriksson, L., Stone-Elander, S., Levander, S., and Ingvar, M. (1995). Brain activation induced by the perceptual maze test: a PET study of cognitive performance. Neuroimage 2, 112-124. 
Gotlib, I. H., Hamilton, J. P., Cooney, R. E., Singh, M. K., Henry, M. L., and Joormann, J. (2010). Neural processing of reward and loss in girls at risk for major depression. Arch. Gen. Psychiatry 67, 380-387.

Gotlib, I. H., and Joormann, J. (2010). Cognition and depression: current status and future directions. Annu. Rev. Clin. Psychol. 6, 285-312.

Grant, M. M., Thase, M. E., and Sweeney, J. A. (2001). Cognitive disturbance in outpatient depressed younger adults: evidence of modest impairment. Biol. Psychiatry 50, 35-43.

Greicius, M. D., Flores, B. H., Menon, V., Glover, G. H., Solvason, H. B., Kenna, H., Reiss, A. L., and Schatzberg, A. F. (2007). Resting-state functional connectivity in major depression: abnormally increased contributions from subgenual cingulate cortex and thalamus. Biol. Psychiatry 62, 429-437.

Greicius, M. D., Krasnow, B., Reiss, A. L., and Menon, V. (2003). Functional connectivity in the resting brain: a network analysis of the default mode hypothesis. Proc. Natl. Acad. Sci. U.S.A. 100, 253-258.

Grimm, S., Ernst, J., Boesiger, P., Schuepbach, D., Hell, D., Boeker, H., and Northoff, G. (2009). Increased self-focus in major depressive disorder is related to neural abnormalities in subcortical-cortical midline structures. Hum. Brain Mapp. 30, 2617-2627.

Hamani, C., Mayberg, H., Stone, S., Laxton, A., Haber, S., and Lozano, A. M. (2011). The subcallosal cingulate gyrus in the context of major depression. Biol. Psychiatry 69, 301-308.

Hamilton, J. P., Chen, G., Thomason, M. E., Schwartz, M. E., and Gotlib, I. H. (2011). Investigating neural primacy in major depressive disorder: multivariate Granger causality analysis of resting-state fMRI time-series data. Mol. Psychiatry 16, 763-772.

Hampson, M., Driesen, N. R., Skudlarski, P., Gore, J. C., and Constable, R. T. (2006). Brain connectivity related to working memory performance. J. Neurosci. 26, 13338-13343.

Harrison, B. J., Pujol, J., ContrerasRodriguez, O., Soriano-Mas, C., Lopez-Sola, M., Deus, J., Ortiz, H., Blanco-Hinojo, L., Alonso, P., Hernandez-Ribas, R., Cardoner, N., and Menchon, J. M. (2011). Taskinduced deactivation from rest extends beyond the default mode brain network. PLoS ONE 6, e22964. doi:10.1371/journal.pone.0022964

Harrison, B. J., Pujol, J., Lopez-Sola, M., Hernandez-Ribas, R., Deus, J., Ortiz, H., Soriano-Mas, C., Yucel, M., Pantelis, C., and Cardoner, N. (2008).
Consistency and functional specialization in the default mode brain network. Proc. Natl. Acad. Sci. U.S.A. 105, 9781-9786.

Harrison, B. J., Yucel, M., Pujol, J., and Pantelis, C. (2007). Task-induced deactivation of midline cortical regions in schizophrenia assessed with fMRI. Schizophr. Res. 91, 82-86.

Harvey, P. O., Fossati, P., Pochon, J. B., Levy, R., Lebastard, G., Lehericy, S., Allilaire, J. F., and Dubois, B. (2005). Cognitive control and brain resources in major depression: an fMRI study using the n-back task. Neuroimage 26, 860-869.

Keedwell, P. A., Andrew, C., Williams, S. C., Brammer, M. J., and Phillips, M. L. (2005). The neural correlates of anhedonia in major depressive disorder. Biol. Psychiatry 58, 843-853.

Kelly, A. M., Di Martino, A., Uddin, L. Q., Shehzad, Z., Gee, D. G., Reiss, P. T., Margulies, D. S., Castellanos, F. X., and Milham, M. P. (2009). Development of anterior cingulate functional connectivity from late childhood to early adulthood. Cereb. Cortex 19, 640-657.

Kyte, Z. A., Goodyer, I. M., and Sahakian, B. J. (2005). Selected executive skills in adolescents with recent first episode major depression. J. Child Psychol. Psychiatry 46, 995-1005.

Lozano, A. M., Mayberg, H. S., Giacobbe, P., Hamani, C., Craddock, R. C., and Kennedy, S. H. (2008). Subcallosal cingulate gyrus deep brain stimulation for treatmentresistant depression. Biol. Psychiatry 64, 461-467.

Margulies, D. S., Kelly, A. M., Uddin, L. Q., Biswal, B. B., Castellanos, F. X., and Milham, M. P. (2007). Mapping the functional connectivity of anterior cingulate cortex. Neuroimage 37, 579-588.

Mason, M. F., Norton, M. I., Van Horn, J. D., Wegner, D. M., Grafton, S. T., and Macrae, C. N. (2007). Wandering minds: the default network and stimulus-independent thought. Science 315, 393-395.

Mayer, J. S., Roebroeck, A., Maurer, K., and Linden, D. E. (2010). Specialization in the default mode: task-induced brain deactivations dissociate between visual working memory and attention. Hum. Brain Mapp. 31, 126-139.

McGorry, P. (1998). Beyond adolescent psychiatry: the logic of a youth mental health model. Aust. N. Z. J. Psychiatry 32, 138-140.

McKiernan, K. A., Kaufman, J. N., Kucera-Thompson, J., and Binder, J. R. (2003). A parametric manipulation of factors affecting taskinduced deactivation in functional neuroimaging. J. Cogn. Neurosci. 15 , 394-408.

Pizzagalli, D. A., Holmes, A. J., Dillon, D. G., Goetz, E. L., Birk, J. L., Bogdan, R., Dougherty, D. D., Iosifescu, D. V., Rauch, S. L., and Fava, M. (2009). Reduced caudate and nucleus accumbens response to rewards in unmedicated individuals with major depressive disorder. Am. J. Psychiatry 166, 702-710.

Price, J. L., and Drevets, W. C. (2010). Neurocircuitry of mood disorders. Neuropsychopharmacology 35, 192-216.

Raichle, M. E., MacLeod, A. M., Snyder, A. Z., Powers, W. J., Gusnard, D. A., and Shulman, G. L. (2001). A default mode of brain function. Proc. Natl. Acad. Sci. U.S.A. 98, 676-682.

Sala-Llonch, R., Pena-Gomez, C. Arenaza-Urquijo, E. M., VidalPineiro, D., Bargallo, N., Junque, C., and Bartres-Faz, D. (2011). Brain connectivity during resting state and subsequent working memory task predicts behavioural performance. Cortex. (in press).

Seeley, W. W., Menon, V., Schatzberg, A. F., Keller, J., Glover, G. H., Kenna, H., Reiss, A. L., and Greicius, M. D. (2007). Dissociable intrinsic connectivity networks for salience processing and executive control. J. Neurosci. 27, 2349-2356.

Sheline, Y. I., Barch, D. M., Price, J. L. Rundle, M. M., Vaishnavi, S. N., Snyder, A. Z., Mintun, M. A., Wang, S., Coalson, R. S., and Raichle, M. E. (2009). The default mode network and self-referential processes in depression. Proc. Natl. Acad. Sci. U.S.A. 106, 1942-1947.

Sheline, Y. I., Price, J. L., Yan, Z. and Mintun, M. A. (2010). Restingstate functional MRI in depression unmasks increased connectivity between networks via the dorsal nexus. Proc. Natl. Acad. Sci. U.S.A. 107, 11020-11025.

Shulman, G. L., Corbetta, M., Buckner, R. L., Fiez, J. A., Miezin, F. M., Raichle, M. E., and Petersen S. E. (1997). Common blood flow changes across visual tasks: II. Decreases in cerebral cortex. J. Cogn. Neurosci. 9, 648-663.

Spreng, R. N., Stevens, W. D., Chamberlain, J. P., Gilmore, A. W., and Schacter, D. L. (2010). Default network activity, coupled with the frontoparietal control network, supports goal-directed cognition. Neuroimage 53, 303-317.

Sridharan, D., Levitin, D. J., and Menon, V. (2008). A critical role for the right fronto-insular cortex in switching between central-executive and default-mode networks.
Proc. Natl. Acad. Sci. U.S.A. 105, 12569-12574.

Supekar, K., Musen, M., and Menon, V. (2009). Development of largescale functional brain networks in children. PLoS Biol. 7, e1000157. doi:10.1371/journal.pbio.1000157

Wagner, A. D., Shannon, B. J., Kahn, I., and Buckner, R. L. (2005). Parietal lobe contributions to episodic memory retrieval. Trends Cogn. Sci. (Regul. Ed.) 9, 445-453.

Wagner, G., Sinsel, E., Sobanski, T., Kohler, S., Marinou, V., Mentzel, H. J., Sauer, H., and Schlosser, R. G. (2006). Cortical inefficiency in patients with unipolar depression: an event-related fMRI study with the Stroop task. Biol. Psychiatry 59, 958-965.

Whitfield-Gabrieli, S., Thermenos, $\mathrm{H}$. W., Milanovic, S., Tsuang, M. T., Faraone, S. V., McCarley, R. W. Shenton, M. E., Green, A. I., NietoCastanon, A., LaViolette, P., Wojcik, J., Gabrieli, J. D., and Seidman, L. J. (2009). Hyperactivity and hyperconnectivity of the default network in schizophrenia and in first-degree relatives of persons with schizophrenia. Proc. Natl. Acad. Sci. U.S.A. 106 , 1279-1284.

Yucel, M., Harrison, B. J., Wood, S. J., Fornito, A., Clarke, K., Wellard, R. M., Cotton, S., and Pantelis, C. (2007). State, trait and biochemical influences on human anterior cingulate function. Neuroimage 34, 1766-1773.

Conflict of Interest Statement: The authors declare that the research was conducted in the absence of any commercial or financial relationships that could be construed as a potential conflict of interest.

Received: 25 November 2011; accepted: 14 February 2012; published online: 28 February 2012

Citation: Davey CG, Yücel $M$, Allen NB and Harrison BJ (2012) Task-related deactivation and functional connectivity of the subgenual cingulate cortex in major depressive disorder. Front. Psychiatry 3:14. doi: 10.3389/fpsyt.2012.00014 This article was submitted to Frontiers in Neuropsychiatric Imaging and Stimulation, a specialty of Frontiers in Psychiatry.

Copyright (c) 2012 Davey, Yücel, Allen and Harrison. This is an open-access article distributed under the terms of the Creative Commons Attribution Non Commercial License, which permits noncommercial use, distribution, and reproduction in other forums, provided the original authors and source are credited. 